\title{
Improving RED Performance during Handovers in Wireless IP Networks
}

\author{
Dagang Li, Johan Theunis, Kristof Sleurs, Jan Potemans, Emmanuel Van Lil, Antoine Van de Capelle \\ Telemic, Dept. of Electrical Engineering-ESAT, Katholieke Universiteit Leuven \\ Kasteelpark Arenberg 10, B-3001 Heverlee, Belgium \\ firstname.lastnamedesat.kuleuven.be
}

\begin{abstract}
Random Early Detection (RED) is a widely used active queue management (AQM) algorithm for congestion avoidance. It monitors the average queue length to detect incipient congestion and notifies the connections of congestion to adjust their sending rate. During a handover, all the active connections of the mobile node will be diverted to the new network and increase the traffic there immediately. Because RED uses an exponential weighted moving average (EWMA) to calculate the queue length, it will be too slow to track this rapid change and fail to react correctly. In this paper we propose a dual RED algorithm to accommodate the handed-over connections quickly while keeping the link utilization high. The improvement is verified with simulations.
\end{abstract}

\section{INTRODUCTION}

Wireless IP networks are becoming more and more popular as the last mile access methods to connect to the global Internet. They provide the access not only to fixed users but also to mobile users who may move out of the coverage of the wireless network during an active connection. In order to reach the Internet again, the mobile user may switch over to another access network that is in range. This is called a handover. When the switching happens between different network technologies, e.g. between Wireless LAN (WLAN), General Packer Radio Service (GPRS) and Universal Mobile Telecommunications System (UMTS), it is called a vertical handover; otherwise it is called a horizontal handover when taking place between networks of the same type.

During a handover, packets to and from the mobile user may be delayed or dropped. For example all the on-the-fly packets will be lost in the old network since the mobile node has already left. One possible solution is to buffer and forward these packets to the new network the mobile user is switched to before the routing is corrected. The exact length of this transition period depends on the type of the handover and the handover techniques used.

On the other hand, handover will affect the other coexisting connections as well. Because all the live connections of the mobile node are diverted from the old network to the new one, the traffic in the new network will increase immediately while the opposite happens to the old network. Since for most wireless networks the capacity is quite limited, this sudden increase may easily cause congestion if the new network does not have enough free bandwidth. In order to solve congestion some of the involving connections should back off and release some occupied bandwidth. The most widely used congestion control mechanisms in the Internet are Transmission Control
Protocol (TCP) and TCP-friendly protocols. With these mechanisms, when congestion in the network is detected, the source node can adjust the sending rate accordingly. A problem with this method is that when several connections detect the congestion at the same time, they will all back off and reduce the total throughput far below the network capacity. This problem is called global synchronization.

Alternatively, the back-off can take place well ahead of time and avoid congestion in the first place. Random Early Detection (RED) [1] is a widely used algorithm for congestion avoidance. It detects incipient congestion by monitoring the average queue length and provides the required notification to the congestion control protocol for pre-emptive back-off. Because connections are not notified at the same time, global synchronization is prevented. In order to accommodate shortterm fluctuation in the network traffic, RED uses an exponential weighted moving average (EWMA) low-pass filter to calculate the average queue length. The side effect is that RED is slow to track rapid traffic change and incapable to react adequately. Unfortunately, this is exactly the case during a handover.

In this paper we propose a dual RED algorithm to improve the performance of RED during a handover. This algorithm provides a method for active congestion control specific for the handover traffic and has minimal changes to the standard RED algorithm. When the handover is finished, it behaves exactly the same way as RED and no overhead is introduced. In the rest of the paper, before explaining the algorithm we first present the background of the problem and some related works in section 2. In section 3 we propose the Dual RED algorithm and discuss its principles. The performance of the algorithm is examined with simulations, which are described in section 4. After the discussions on the simulation results, section 5 concludes the paper.

\section{PRoblems OF RED with HANDOVER}

The problem of RED with handover is that RED can not cope with the abrupt change in the network load cause by handover. Therefore, this problem can be tackled from two directions: we can make RED faster in detecting the handover caused change, or we reduce the impact of the handover on the traffic in the new network.

\section{A. The RED Algorithm}

RED avoids congestion by keeping the queue from getting full. With a smaller queue, the queuing delay is lower. At the 
same time, because the queue is never full, congestion will not happen and therefore the average throughput is high.

The target of RED is to keep the average queue length within a minimum threshold $\min _{t h}$ and a maximum one $\max _{t h}$. It uses EWMA [1] to calculate the average queue length:

$$
a v g \leftarrow\left(1-w_{q}\right) a v g+w_{q} \cdot q
$$

where $w_{q}$ is the weight determining the time constant of the filter, and $q$ is the current queue length. The calculated $a v g$ is then compared to the two thresholds and the following actions are performed according to the comparison:

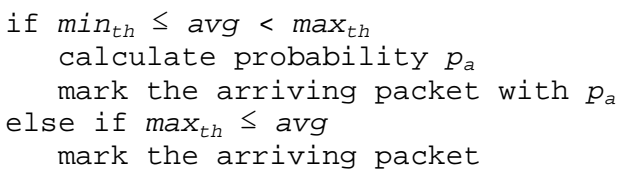

If marked packets are in fact dropped or if all source nodes are cooperative, this ensures the avg does not significantly exceed $\max _{t h}$. The packet marking probability is represented by the curve in Figure 1. $\max _{p}$ is another important parameter of RED which is the maximum marking probability before every arriving packet is marked. limit $_{q}$ is the size of the buffer.

Multiple REDs can be used in a related way. For example they can resident in the same queue as in RIO (RED with In and Out) [4], or each in a separate queue but share a common buffer [5].

\section{B. The RED Problem with Handover}

The traffic increase caused by a handover is normally the sum of all the active connections of the mobile user right before the handover. In most cases these connections should have settled down to a steady rate and the sum of which is certainly larger than zero. Therefore the traffic increase in the new network is indeed a step change and will disturb the network status.

1) Network is lightly utilized: In this case avg is normally below $\min _{t h}$ and arriving packets are not marked. The step change in the traffic may build up the queue quickly but because avg grows slowly, no packet gets marked to control the queue growth; when avg finally goes above $\min _{t h}$ and RED starts marking packets, since the current queue length is very high, avg will stay above $\min _{t h}$ for a longer time and some packets will get marked unnecessarily. The excessive marking drives down the throughput too much and the link utilization becomes low, as shown in Figure 5 and Table 2.

2) Network is moderately utilized: In this case avg is between $\min _{t h}$ and $\max _{t h}$ and arriving packets are selectively marked to keep the average queue length in range. Because RED can not follow the step change, marking rate will not be sufficient and the queue size can grow beyond $\max _{t h}$ or even limit $_{q}$ and cause global synchronization, which severely degrades the link utilization as shown in Figure 6 and Table 2.

3) In case of congestion: Since all the arriving packets are already marked, the handed-over traffic will at the best make recovery more difficult. In this paper this extreme case is not considered.

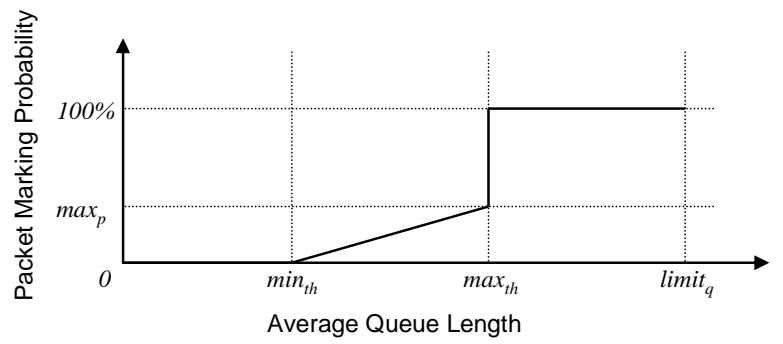

Fig. 1 RED packet marking probability

\section{Related Works}

Only a few papers in the literatures target directly at the problem studied in this paper. Roh et al. and Hur et al. have studied this problem with TCP and proposed Implicit Priority Forwarding (IPF) [2] and Priority Forwarding (PF) [3] to improve the performance and link utilization. Unfortunately the gain is quite limited comparing to the standard RED.

The reason in IPF is that it excludes the forwarded packets (buffer and forward is always assumed, because it minimizes packet loss during a handover) from the avg calculation in the hope not to drive avg abruptly above $\max _{\text {th }}$ so as to avoid global synchronization. Unfortunately, after the forwarding is finished, the traffic of the mobile node will still be included in the calculation of the avg, therefore the avg tracking is just delayed, which in turn delays the reaction of RED; since the forwarded packets are never marked by RED, the situation gets even worse because the queue also builds up faster[3].

In $\mathrm{PF}$, forwarded packets are put into a separate queue which has a higher priority than the RED. They are never marked and get forwarded before the packets in the RED. It is claimed that by doing so the packets for the RED will arrive at a slower pace, therefore the RED queue builds up slower and less packets get marked. But like in IPF, because no forwarded packets are marked, the rate of the mobile node's traffic is kept at the same level; at the same time, since the marking of the packets from RED is not increased, their sending rate is not reduced either (the temporary slow down caused by higher queuing delay does not affect the congestion window of TCP, therefore when the priority queue is gone, the sending rate is resumed). So the problem basically remains when the forwarding completes.

The problem of these two proposals is that they do not actively use packet marking to adjust the traffic for handover but rely on the marking algorithm from the standard RED. Therefore the performance gain is limited. Nevertheless, they still give us the hint that the forwarded packets are the best object for RED to apply differentiated handling to the handedover traffic of the mobile node.

\section{DUAL RED ALGORITHM}

In this section we present the design considerations and the principles of our proposed Dual RED algorithm.

From the discussion in the previous sections, we believe that a new method is needed for RED to deal with the abrupt changes in the network caused by handovers. This method 


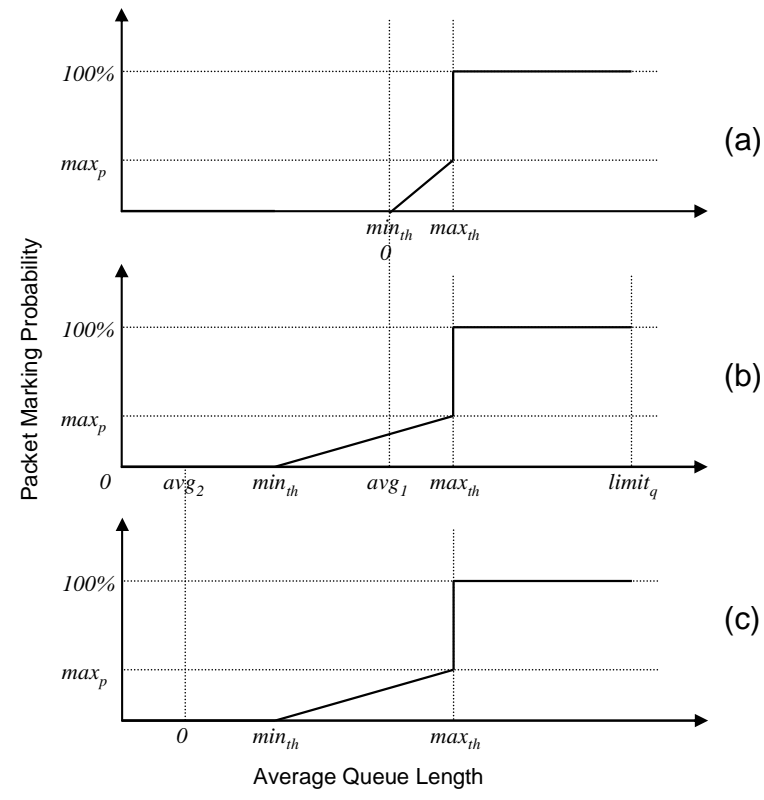

Fig. 2 Packet marking probability of dual RED

should actively assist the RED to adapt to the new network condition without performance degradation. It should be simple and effective and require little modification to the standard RED, so normal RED behavior is not disturbed by the handover. It should also be light-weighted and cause minimum overhead when no handover is happening.

Based on these requirements, we decide to design a kind of add-on to the standard RED, which does not modify the RED and is not invoked unless needed. The proposed mechanism is called dual RED, because in this mechanism the original RED (oRED) is kept unchanged and an add-on RED (aRED) is only used temporarily during a handover to handle the handed-over traffic. The idea is to differentiate handed-over traffic from short burst traffic which should be tolerated by RED, and adjust the former adequately. The behavior of the aRED is closely coupled to both the oRED settings and the network condition, therefore tuning aRED adequately can improve the overall performance during a handover.

\section{Queue Structure}

The structure of dual RED is similar to RIO proposed in [4], since both of them have more than one RED profile but only one queue for all the arriving packets. In dual RED, the aRED is only applied on the forwarded packets, while the oRED marks all other packets based on the measurement of the whole queue. Comparing to RIO, oRED can be thought as the profile for the out packets and aRED for the in packets which, in our case, are the forwarded packets. Note here that when the packets for the mobile node are not forwarded any more but routed directly to the new network, they will be handled not by aRED any more buy by the oRED. In this way, oRED takes over the traffic of the mobile node after the handover is completed, and aRED is not used thereafter. The purpose of aRED is to assist the oRED to settle down fairly with the extra traffic of the handed-over mobile node.
We choose a single queue structure to accommodate both oRED and aRED, so oRED always has a complete view of all the arriving packets, including the forwarded packets during a handover. On the other hand, like in PF and IPF we treat the forwarded packets separately in order to change their impact on the new network. Here we use the aRED for this purpose. Because all the packets are in the same queue, the change in the forwarded traffic will also be seen by the oRED, thus creating a way for the aRED to influence the oRED.

With a separate RED dedicated to the forwarded packets, we can make its avg track closely the dynamics of the handedover traffic. Unlike temporary burst, handed-over traffic will cause long-lasting changes to the network status, therefore its queuing dynamics is a quick reflection of the trend and should not be filtered out. A separate RED also provides a direct way to manipulate the handed-over traffic specifically.

\section{E. Parameter Settings}

Although the structure of dual RED and RIO are quite similar, their working principles are very different.

First, in RIO, in packets are designed to have a higher priority and a smaller probability to get marked, while out packets are served in a more opportunistic way; whereas in dual RED, there is no intension to provide better service to the forwarded packets. Second, the RED profile for in and out packets are independent in RIO, but in dual RED the parameters of aRED depends on the settings of the oRED and the network condition. This creates a way of interaction in the opposite direction for the oRED to influence the aRED.

More specifically, the thresholds $\min _{t h}$ and $\max _{t h}$, the packet marking probability $\max _{p}$ and the weight $w_{q}$ of the aRED are calculated as below in the run-time:

$$
\begin{aligned}
& \max _{t h}^{\mathrm{aRED}}=\max _{t h}^{\mathrm{oRED}}-a v g^{\text {oRED }} \\
& \min _{t h}^{\mathrm{aRED}}=\max \left(0, \min _{t h}^{o R E D}-a v g^{\text {oRED }}\right) \\
& \max _{p}^{\mathrm{aRED}}=\max _{p}^{o R E D} \\
& w_{q}^{\mathrm{aRED}}=1-\exp \left(-1 / R_{a v g}\right)
\end{aligned}
$$

The relation between the packet marking probability of oRED and aRED is also shown in Figure 2, where 2(b) is for the oRED. The avg of the oRED is a measurement of the network condition and the higher it is, the more crowded the network. Figure 2(a) and 2(c) shows the parameters of the aRED when $a v g^{\text {oRED }}$ is in different region.

Equation (2) and (3) ensure that the aggregated queue length, with the forwarded packets included, is still bounded by the thresholds of the oRED, so no global synchronization will happen. Equation (4) gives the same marking probability for all the packets when $\max _{t h}{ }^{\text {oRED }}$ is reached globally. On the other hand, the packet marking probability of the aRED always starts from $0 \%$ no matter where the $a v g^{\text {oRED }}$ is, as shown in Figure 2(a) and 2(c). This is to make sure that sufficient forwarded packets are accepted into the queue in the beginning. In a later time, if the forwarded packets contribute too much to the queue growth, aRED will kick in very soon.

$R_{\text {avg }}$ in equation (5) is the "time constant" [9] of the $a v g^{\text {aRED }}$ estimator. The smaller it is, the faster the estimator is to 
follow the instantaneous change in the queue length. It should be set to ensure that the reaction time of the estimator is in the order of the handover delay. Because $a v g^{\text {aRED }}$ follows the queue length growth faster than $a v g^{\mathrm{ORED}}$, aRED also reacts faster than oRED, therefore the queue building-up problem will be handled sooner than in the traditional single RED case.

The pseudo-code of dual RED is shown in Figure 3.

set up forwarding tunnel

$$
\text { calculate } w_{q}
$$

for each packet arrival

calculate the average total queue size $a v g^{O R E D}$

calculate $\min _{t h}{ }^{a R E D}$ and $\max _{t h}{ }^{a R E D}$

if it is a forwarded packet

calculate the average queue size avg ${ }^{a R E D}$

if $\min _{t h}{ }^{a R E D} \leq a v g^{a R E D}<\max _{t h}{ }^{a R E D}$

calculate probability $p_{a}{ }^{a R E D}$

mark the arriving packet with $p_{a}^{a R E D}$

else if $\max _{t h}{ }^{a R E D} \leq a v g^{a R E D}$

mark the arriving packet

else

if $\min _{t h}{ }^{O R E D} \leq \mathrm{aVg}^{O R E D}<\max { }^{O R E D}$

calculate probability $p_{a}{ }^{O R E D}$

mark the arriving packet with $p_{a}{ }^{\text {ORED }}$

else if $\max _{t h}{ }^{O R E D} \leq a v g^{O R E D}$

mark the arriving packet

Fig. 4 Dual RED algorithm

We do not claim here that the setting of the parameters in this paper is optimal. But it is already sufficient to show the idea of dual RED that by intentionally manipulating the forwarded packets, the performance of a RED enabled network during a handover can be improved and high link utilization can be achieved.

\section{PERformance EVAluation}

In this section we will present our simulation results to evaluate the performance of the proposed dual RED algorithm in different traffic conditions. We also show the performance of standard RED under the same network condition alongside for a comparison. All the simulations are done in ns-2 [10].

Since we are more interested in the queue management behavior during handovers in a general wireless environment, the wireless connections in the simulations are generalized as a bandwidth-limited link shared by all the served users. In order to focus on the effect of the handover, we assume wireless transmission errors are handled by the lower layers and cause no packet loss; because handovers normally take a short time, we also assume the capacity of the wireless link to be constant during the handover. Therefore the simple network in Figure 4 is used for all the simulations, where the bandwidth-limited links of AR1-BS1 and AR2-BS2 represent the characteristics of wireless connections.

A handover takes place when the mobile node (MN) changes its attachment from BS2 to BS1. After the handover all the traffic between the correspondent node $(\mathrm{CN})$ and the $\mathrm{MN}$ will be handed-over from R2-AR2-BS2 to R2-AR1-BS1. As explained before, we use buffer and forward to minimize packet loss during the handover, therefore the packets for the $\mathrm{MN}$ that are still routed to AR2 will be forwarded to AR1, thus take the route R2-AR2-AR1-BS1. After the handover is completed, all the packets for the MN will be routed directly to AR1 from R2. In these simulations reverse tunneling [8] is

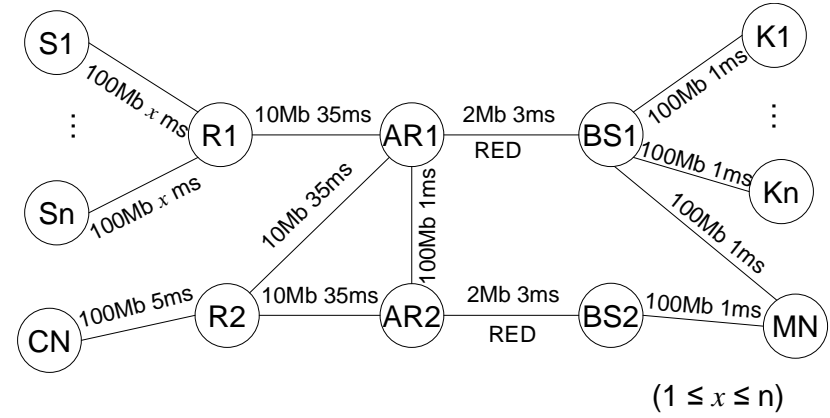

Fig. 3 Simulation network

also used, so traffic of the opposite direction always takes the reverse route of the forwarding traffic. If we refer to Mobile IP [7] here, R2 can be regarded as the Home Agent, and Foreign Agents resident in the two access routers.

The traffic between the $\mathrm{S}$ nodes and the $\mathrm{K}$ nodes will be the background load on the link between AR1 and BS1, where either Dual RED or standard RED is applied. All the other links have DropTail queues. In the simulations, Explicit Congestion Notification (ECN) [6] is used for packet marking. All traffic is well behaved, using TCP to control the sending rate according to the ECN marking. Both bulk data (FTP) traffic and busty (WEB) traffic are present in the simulations.

For the settings of the RED, the popular thumb rules from [9] are used. In our simulations the $\mathrm{min}_{t h}$ is increased from 5 to 15 to accommodate the busty WEB traffic. The settings used in the simulations are shown in Figure 4 and Table 1. In our settings the handover takes $500 \mathrm{~ms}$ to complete.

TABLE I

SIMULATION PARAMETERS

\begin{tabular}{|l|l|l|l|l|l|}
\hline \multirow{2}{*}{ RED } & $\boldsymbol{m i n}_{\text {th }}$ & max $_{\text {th }}$ & max $_{\boldsymbol{p}}$ & $\boldsymbol{w}_{\boldsymbol{q}}$ & $\boldsymbol{R}_{\text {avg }}$ \\
\cline { 2 - 6 } & 15 & 45 & 0.1 & 0.002 & 10 \\
\hline \multirow{3}{*}{ FTP } & type & packet size & web burst \\
\cline { 2 - 6 } & SAck & 1000 byte & 10 packets \\
\hline \multirow{2}{*}{ Simulation } & total time & HO starts & HO ends \\
\cline { 2 - 6 } & $200 \mathrm{~s}$ & $100 \mathrm{~s}$ & $100.5 \mathrm{~s}$ \\
\hline
\end{tabular}

Because of the space limit, we only present the simulation results in the two scenarios discussed in section 2 to compare dual RED with the standard RED.

\section{A. The Lightly Loaded Network Case}

In this scenario the link AR1-BS1 is lightly loaded when the handover happens. There are in total 3 TCP connections between the $\mathrm{S}$ nodes and the K nodes, with $\mathrm{RTT}$ ranging from $80 \mathrm{~ms}$ to $82 \mathrm{~ms}$. The FTP traffic is limited by the congestion window size of 15 . There is also random web burst traffic happening at a frequency of 2 per second per direction. The $\mathrm{MN}$ has a long-living FTP connection with the $\mathrm{CN}$. The simulation result is shown in Figure 5.

From Figure 5 we can see that handover causes queue increase with both techniques, and drive the average queue length cross the $\min _{t h}$ from below. Dual RED clearly reduces the queue building-up and causes less fluctuation in the average queue length, whereas in standard RED, the fluctuation leads to the alternation between higher packet marking rate and lower queue occupation. 

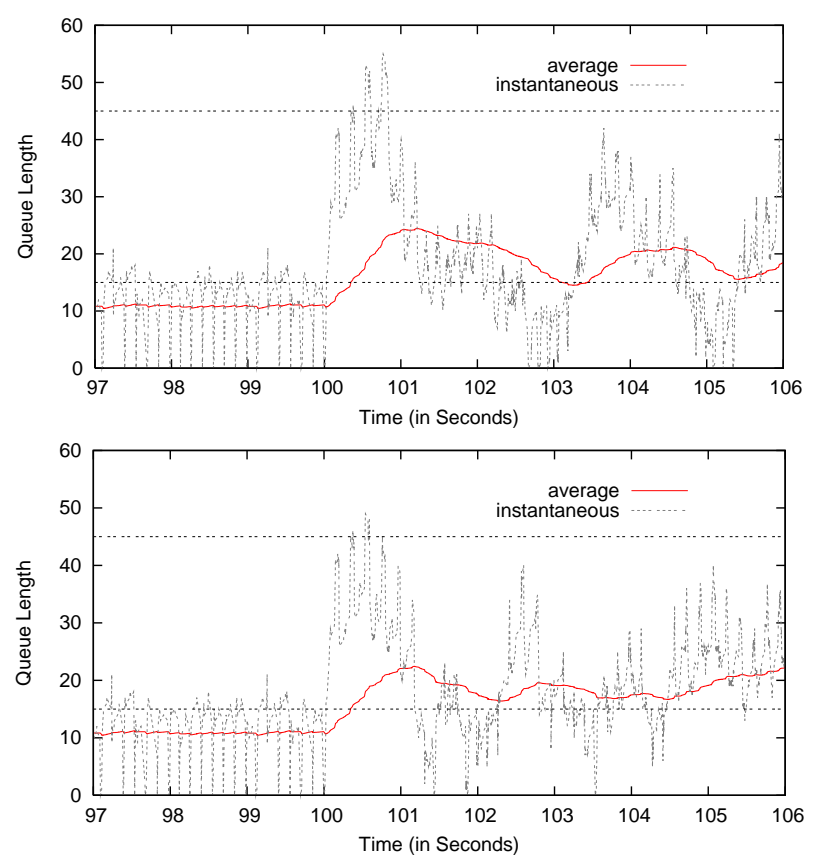

Fig. 5 Handover to a light network with RED (upper) and dual RED

The spikes in the figures are caused by the WEB traffic.

\section{B. The Moderately Loaded Network Case}

In this scenario the number of the TCP connections is increased to 7, both directions included, with RTT from $80 \mathrm{~ms}$ to $88 \mathrm{~ms}$. The web traffic is kept the same as in the previous scenario. The FTP congestion window size is enlarged to 64 packets, so the transmission rate is now limited by the bottleneck in the network. The result is shown in Figure 6.

In this case, RED already works in the congestion avoidance region before the handover. The handed-over traffic causes a sudden increase in the average queue length, and drives the standard RED above max $_{\text {th }}$ which triggers global synchronization. Dual RED restricts the average queue length below $\max _{\text {th }}$ through run-time adaptation, which limits the throughput degradation during the handover.

\section{The Moderately Loaded Network Case}

The link utilization of dual RED and standard RED in the above two scenarios is listed in Table 2. The results are based on 10 runs of each scenario where handover takes place at random moment. The throughput is calculated over a 5 -second period from the beginning of the handover. We can see that dual RED achieves higher average throughput and lower standard deviation (STD) in both scenarios. This means that the performance of dual RED is also more robust to the transitional network condition at the time of the handovers.

TABLE II

PERFORMANCE COMPARISON

\begin{tabular}{lllll}
\hline Throughput \% & dual-1 & RED-1 & dual-2 & RED-2 \\
Average & 99.4 & 98.8 & 94.9 & 94.1 \\
STD & 0.36 & 0.61 & 1.93 & 3.16 \\
\hline
\end{tabular}
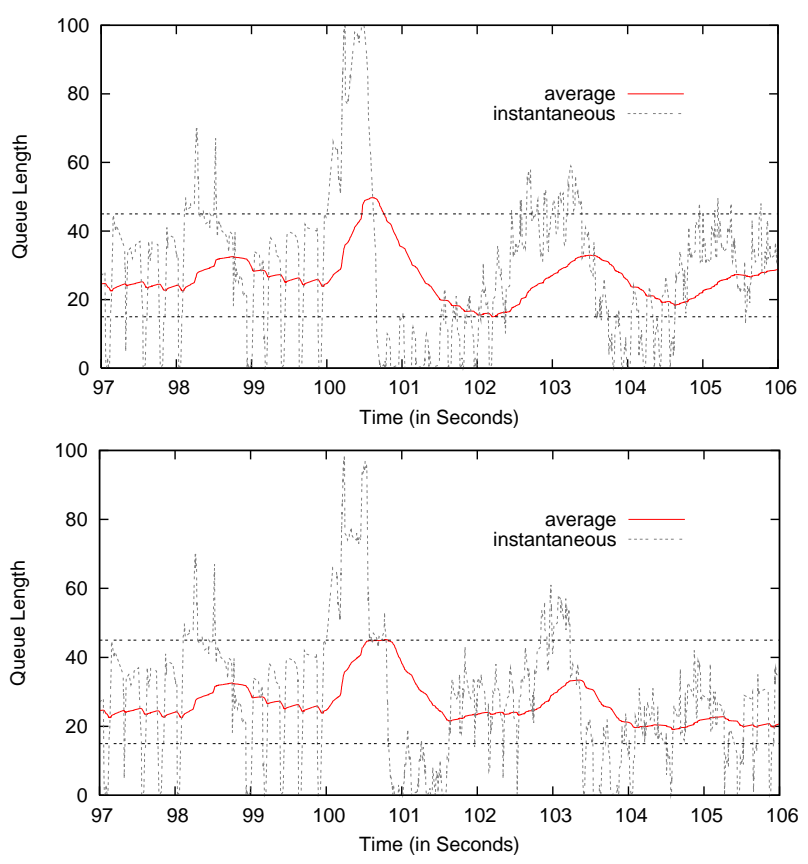

Fig. 6 Handover to a crowded network with RED (upper) and dual RED

\section{CONCLUSIONS}

In this paper we have proposed dual RED to improve the performance of RED during handover of wireless IP networks. During a handover, an add-on RED is temporarily added to the standard RED queue to regulate the forwarded packets of the mobile node, so that the handed-over traffic is quickly adapted to the condition of the new network without causing global synchronization or degradation in the link utilization.

\section{REFERENCES}

[1] S. Floyd and V. Jacobson, "Random Early Detection Gateways for Congestion Avoidance", IEEE/ACM Transactions on Networking, 1(4):397-413, Aug. 1993.

[2] Y. S. Roh, K. Hur, D.-S. Eom, Y. Lee and K. H. Tchah, "TCP Performance Enhancement by Implicit Priority Forwarding (IPF) Packet Buffering Scheme for Mobile IP based Networks", Journal of Communication and Networks, vol. 7, no. 3, pp. 367-376, 2005.

[3] K. Hur, D.-S. Eom, Y.W. Lee, J.H. Lee and S. Kang, "Priority Forwarding for Improving the TCP Performance in Mobile IP based Networks with Packet Buffering", Computer Communications 30(2007), pp. 1337-1349, Elsevier, 2007.

[4] D. D. Clark and W. Fang, "Explicit Allocation of Best-Effort Packet Delivery Service", IEEE/ACM Transactions on Networking, Vol. 6, No. 4, pp. 362-373, August 1998.

[5] F. Agharebparast and V. C. M. Leung, "Improving the Performance of RED Deployment on a Class Based Queue with Shared Buffers", in Proc. IEEE Globecom'01, pp. 2363-2367, San Antonio, TX, Nov. 2001

[6] S. Floyd, "TCP and Explicit Congestion Notification", ACM Computer Communication Review, vol. 24, no. 5, pp. 10-23, October 1994.

[7] C. Perkins, Ed. "IP Mobility Support for IPv4", RFC 3344, IETF, August 2002

[8] G. Montenegro, Ed. "Reverse Tunneling for Mobile IP, revised", RFC 3024, IETF, January 2001

[9] S. Floyd. "RED: Discussions of Setting Parameters" [Online]. Available: http://www.aciri.org/floyd/REDparameters.txt

[10] The Network Simulator - ns-2, website. [Online]. Available: http://www.isi.edu/nsnam/ns 